\title{
Epoxy Nanocomposites Based on Silylated Montmorillonite: Effect of the Coupling Agents Structure on the Mechanical Properties
}

\author{
E. Amendola*, ${ }^{*}$, A.M. Scamardella ${ }^{1,2}$, G. Callegaro ${ }^{1}$, M. Lavorgna ${ }^{1}$, F. Piscitelli ${ }^{2}$ and V. Romeo ${ }^{1}$ \\ ${ }^{I}$ Institute of Composite and Biomedical Materials, CNR - Italy's National Council of Research, P.le Tecchio 80, 80125 \\ Naples, Italy \\ ${ }^{2}$ Department of Materials and Production Engineering, University of Naples Federico II, P.le Tecchio 80, 80125 \\ Naples, Italy
}

\begin{abstract}
MMT clay-based polymer nanocomposites have been widely studied because of their low cost and unique characteristics, as well as their applications in commercial sectors. The aim of this work was to evaluate the effect of MMT silylated by 3-aminopropyltriethoxysilane and N-(2-aminoethyl)-3-aminopropyltrimethoxysilane on the mechanical behaviour of MMT/epoxy nanocomposites by evaluation of dynamical mechanical analysis and nanoindentation technique. The silicate clay layers were dispersed in the epoxy matrix by sonication process obtaining the intercalation of the epoxy resin inside the inorganic galleries of MMT. The results showed that the MMT modified with N-(2-aminoethyl)-3aminopropyltrimethoxysilane, which is characterized by the longer alkyl functional group, exhibits an higher compatibility with polymer matrix and improved mechanical properties.
\end{abstract}

Keywords: Organo-clay, MMT, aminosilane, coupling agent, epoxy nanocomposites.

\section{INTRODUCTION}

Nanocomposite materials have evoked intensive and extensive academic and industrial research activities in the recent decade due to their unique properties resulted from the integration of the characteristics of organic and inorganic component at the nanometric scale. The ultimate physical properties of nanocomposites depend strongly on the particle size, shape, degree of dispersion, and the interactions occurring at the interface between particles and polymer matrix [1]. However, when the dimension of inorganic additives reduces to nanoscale size, the difficulty of homogeneously dispersing inorganic parts in the organic matrix is dramatically increased, owing to the lack of affinity between the interfacial contact area of inorganic fillers and organic polymers. The functionalization of inorganic fillers by an organic agent was usually an effective way to enhance compatibility between two phases [2].

Among the several nanoparticles, montmorillonite (MMT) is widely used to produced nanocomposites because it is a natural and cheap material and also it exhibits a peculiar layered structure which is suitable to enhance the thermal stability, fire resistance, gas permeability and mechanical properties of polymeric materials.

Unfortunately, the hydrophilic characteristic of the clay due to the presence of hydroxyl groups reduces its compatibility with most interesting polymers, such as epoxy resins. Several methods have been studied to modify or reduce the hydrophilicity of the internal galleries of the layered silicates and increase the adhesion with the organic matter. At the

*Address correspondence to this author at the Institute of Composite and Biomedical Materials, CNR - Italy's National Council of Research, P.le Tecchio 80, 80125 Naples, Italy; Tel: (+)39 081 7758827: Fax: (+)39 081 7758850; E-mail: amendola@unina.it moment, the ion exchange reactions between alkyl ammonium salts and the $\mathrm{Na}^{+}, \mathrm{K}^{+}$or $\mathrm{Ca}^{++}$ions present between the platelets of MMT is considered being the most attractive approach. As an alternative, the silylation of the clay surfaces with organic silanes has been proposed as an interesting method to tailor the surface properties of MMT layers and improve the interaction with the epoxy resins [3].

In this study, the effect of the chemical structure of coupling agents on the mechanical properties of epoxy nanocomposites has been investigated. In particular, the silylation of MMT has been performed by using 3-aminopropyltriethoxysilane and $\mathrm{N}$-(2-aminoethyl)-3-aminopropyltrimethoxysilane, which are characterized by a different structure of the organic moieties [4-6]. The nanocomposites have been produced by sonication and the resulting materials can be classified as "intercalated", where the epoxy resin is located in between the inorganic silicate galleries of the MMT clay. Mechanical properties were investigated by dynamic mechanical analysis, and nanomechanical parameters as hardness and creep behaviour were determinated by using nanoindentation technique.

\section{EXPERIMENTAL}

\section{Materials}

The sodium montmorillonite (denoted as Na-MMT), with cationic exchange capacity (CEC) of $91 \mathrm{meq} / 100 \mathrm{~g}$, was purchased from Southern Clay Products Inc., USA. Both the 3aminopropyltriethoxysilane (A1100) and N-(2-aminoethyl)3-aminopropyltrimethoxysilane (A1120) were obtained from GE Advanced Materials. Glycerol, diglycidyl ether of bisphenol-A (DGEBA) with equivalent weight (EEW) 187 $\mathrm{g} / \mathrm{mol}$ eq and meta-xylylenediamine (MXDA) were purchased from Sigma Aldrich. All materials were used as received. 


\section{Preparation of Modified Clay}

Na-MMT has been used after being dried for $1 \mathrm{~h}$ at $90^{\circ} \mathrm{C}$ under vacuum condition. The grafting reaction was carried out by mixing in glycerol (94.0 g) Na-MMT (3.0 g) and aminosilanes $(3.0 \mathrm{~g})$ and stirring at $80^{\circ} \mathrm{C}$ for $3 \mathrm{~h}$. Then the product was separated by centrifugation, washed in distilled water and dried for $3 \mathrm{~h}$ at $80^{\circ} \mathrm{C}$ in vacuum condition [7].

Montmorillonite (MMT) and modified montmorillonite with (1) 3-aminopropyltriethoxysilane and (2) N-(3(trimethoxysilyl)propyl)ethylene-diamine were used for nanocomposites preparation.

\section{Preparation of Nanocomposites}

The clay amount was first added to the DGEBA and mixed by a Thinky planetary vacuum mixer, for 2 minutes at $2000 \mathrm{rpm}$. The mixture was then sonicated for 30 minutes. Next, hardener was added and the solution was degassed for 4 minutes, and finally placed in moulds and cured according to the following thermal cycle: $30^{\circ} \mathrm{C}$ for $1 \mathrm{~h}, 60^{\circ} \mathrm{C}$ for $1 \mathrm{~h}$, $90^{\circ} \mathrm{C}$ for $1 \mathrm{~h}, 100^{\circ} \mathrm{C}$ for $1 \mathrm{~h}, 120^{\circ} \mathrm{C}$ for $1 \mathrm{~h}$ and $150^{\circ} \mathrm{C}$ for $1 \mathrm{~h}$.

The samples made of unmodified MMT were abbreviated as Na-MMT and the samples made of modified MMT were abbreviated as: (1) MMT-A1100, (2) MMT-A1120. Composites with 1 and $3 \%$ by weight of inorganic filler were prepared. The amount of silylated MMT filler has been calculated by taking into account the actual content of inorganic phase (i.e. MMT without the organic moieties) through the evaluation of the organic content due to the presence of coupling agents present in the filler [8].

\section{Nanocomposite Characterization Methods}

Differential scanning calorimetry was carried out to verify complete cure for all composites using a TA Instruments DSC Q1000. The tests were recorded at heating rate of $10^{\circ} \mathrm{C} / \mathrm{min}$ in a temperature range from $0^{\circ} \mathrm{C}$ to $200^{\circ} \mathrm{C}$.

Dynamic-mechanical measurements were performed by a TA Instruments DMA Q800, by a single cantilever measuring geometry and varying the temperature at a heating rate of $3^{\circ} \mathrm{C} / \mathrm{min}$, from $0^{\circ} \mathrm{C}$ to $180^{\circ} \mathrm{C}$. The experiments were carried out at a frequency of $1 \mathrm{~Hz}$ and $25 \mu \mathrm{m}$ strain amplitude.

The indentation experiments were performed with a Micro Materials Ltd NanoTest ${ }^{\mathrm{TM}}$ Platform. A Berkovich diamond tip with specified geometry (three-side pyramidal) is driven to indent the surface of sample. In this work the nanoindentation tests was conducted at a maximum load of 100 $\mathrm{mN}$, with an initial load of $0.02 \mathrm{mN}$. The load was then hold at maximum for $60 \mathrm{~s}$ in order to separate the elastic and plastic behavior of the materials to determine mechanical parameters as elastic modulus, hardness and creep behavior. At least 20 indents were performed on each sample and the distance between the indentations was $100 \mathrm{~nm}$ to avoid any interaction. In an indentation test the parameters (hardness and elastic modulus) are evaluated according to the Oliver and Pharr method [9].

\section{RESULTS AND DISCUSSION}

DSC results (not reported for sake of brevity) confirm the absence of residual reactivity and show a complete cure of cross-linking reactions of epoxy resins.
Dynamic-mechanical spectra, in the form of plots of storage modulus $\mathrm{E}^{\prime}$ and $\tan \delta$ as function of temperature, are showed in Fig. (1), for the pure epoxy resin and for its nanocomposites containing $1 \% \mathrm{wt}$ of unmodified and modified clay. Table 1 summarizes the elastic modulus and $T_{g}$ values of epoxy-composites. The $\mathrm{T}_{\mathrm{g}}$ values are taken at the maximum of $\tan \delta$ curves.

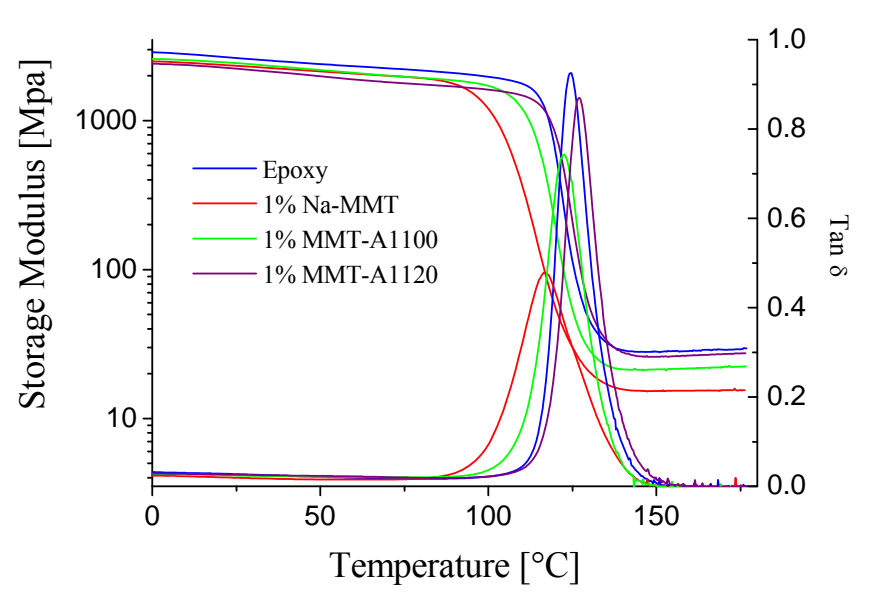

Fig. (1). Storage Modulus and Tan $\delta$ as a function of temperature for epoxy resin and its nanocomposites at $1 \% \mathrm{wt}$ of Na-MMT, MMT-A1100 and MMT-A1120.

The presence of unmodified and modified clay does not significantly affect the $\mathrm{T}_{\mathrm{g}}$ value of the nanocomposites. Nevertheless a small increase is shown for the 1\%MMT-A1120 composite, as showed in Table 1. This is probably due to the higher extent of intercalation of epoxy resins in comparison to the materials prepared by A1000; the dimension of the silicate galleries of silylated MMT increases with the length of the organic moieties of the aminosilanes used for the silylation reaction [10].

The elastic modulus values $\left(25^{\circ} \mathrm{C}\right)$ of Na-MMT, MMTA1100 and MMT-A1120, at different weight percentages, are somewhat comparable to that of neat epoxy, accordingly with $\mathrm{T}_{\mathrm{g}}$ results.

Table 1. Storage Modulus and $T_{g}$ Values of Epoxy-Clay Nanocomposites

\begin{tabular}{|c|c|c|}
\hline Samples & Storage Modulus $^{1}$ [GPa] & $\left.\mathbf{T}_{\mathbf{g}}{ }{ }^{\circ} \mathbf{C}\right]$ \\
\hline \hline Epoxy resin & 2,6 & 124 \\
\hline $1 \%$ Na-MMT & 2,4 & 118 \\
\hline $1 \%$ MMT-A1100 & 2,5 & 123 \\
\hline $1 \%$ MMT-A1120 & 2,3 & 127 \\
\hline $3 \%$ Na-MMT & 2,6 & 104 \\
\hline $3 \%$ MMT-A1100 & 2,3 & 117 \\
\hline $3 \%$ MMT-A1120 & 2,3 & 122 \\
\hline
\end{tabular}

${ }^{1}$ The values are calculated at $25^{\circ} \mathrm{C}$, from DMA tests.

Fig. (2) shows the loading-hold-unloading profiles of epoxy and its nanocomposites under a maximum load of 100 


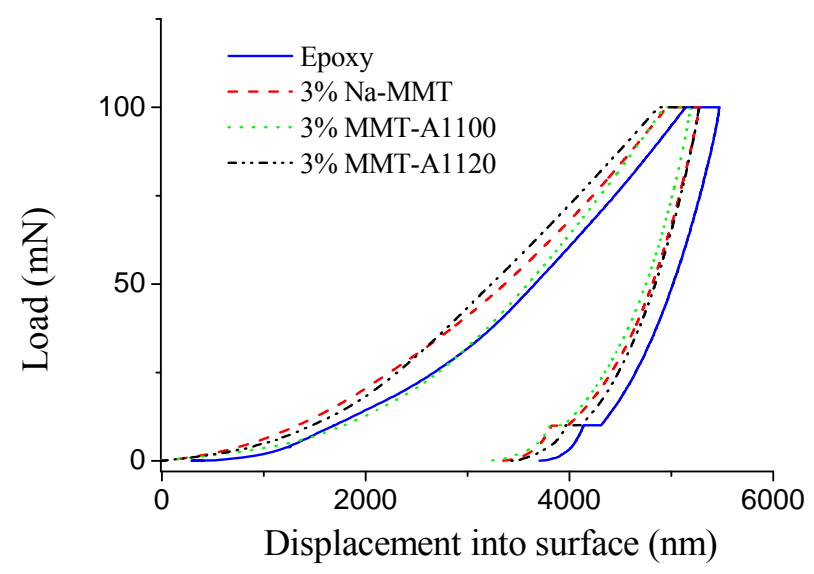

Fig. (2). Load-displacement relation of neat epoxy and its nanocomposites.

$\mathrm{mN}$. It is found that, with the same loading condition, neat epoxy resin (solid curve) possesses the lowest resistance to indentation force and the highest maximum indentation depth at $100 \mathrm{mN}$, both before and after the maximum hold segment. It is also noticed that the slope of the load- unloading curve and hence the calculated elastic modulus. According to Zheng et al. [12] elastic modulus measured by depth sensing indentation are invariantly higher than tensile test by a value of $10-20 \%$. However it is worth noting that the increment of the elastic modulus for the samples with silylated MMT filler can be ascribed to the presence of intercalated tactoids which affect the penetration and displacement of the diamond tip of the indentation device.

\section{CONCLUSION}

In the present study, the mechanical properties of the epoxy-organoclay nanocomposites (1-3\% wt of Na-MMT, MMT-A1100 and MMT-A1120), prepared by sonication technique, have been studied by using the dynamicalmechanical analysis and nanoindentation analysis. The use of sonication has enabled well-dispersed epoxy-clay composites to be prepared, where the epoxy resin results intercalated in the silicate MMT galleries. The elastic modulus of nanocomposites are almost the same compared with the neat epoxy but, the Tg values result slightly increased, in particular for the montmorillonite silylated with the aminosilane characterized by the organic moieties with the longer alkyl backbone (i.e. A1120 coupling agent). It exhibits probably an higher compatibility with epoxy polymer matrix. The addition of clay nanofiller reduces the creep resistance, which could be attributed to the effect of the inorganic filler

Table 2. Mechanical Parameters from Indentation Test

\begin{tabular}{|c|c|c|c|}
\hline Samples & Elastic Modulus [GPa] & Creep displacement at 60 s [nm] & Hardness [GPa] \\
\hline \hline Epoxy resin & $4.01 \pm 0.333$ & $348.90 \pm 12.40$ & $0.145 \pm 0.055$ \\
\hline $3 \%$ Na-MMT & $4.10 \pm 0.195$ & $297.18 \pm 15.46$ & $0.220 \pm 0.0162$ \\
\hline $3 \%$ MMT-A1100 & $4.27 \pm 0.345$ & $298.83 \pm 13.73$ & $0.193 \pm 0.067$ \\
\hline $3 \%$ MMT-A1120 & $4.34 \pm 0.432$ & $336.65 \pm 11.67$ & $0.162 \pm 0.0519$ \\
\hline
\end{tabular}

dispalcement curve at the initial unloading portion increases with the length of aminosilanes. In fact, this slope corresponds to a moderate increase of materials' elastic modulus.

The maximum holding load segment in the load-holdunload cycle is used to explore the creep behavior of the epoxy and its nanocomposites. It is reasonable to find that clay addition little increases the creep resistance, as showed in Table 2.

The decrease in the creep resistance, as well as the $\mathrm{Tg}$ values, could be due to the plasticizing effect from the organic modifier within the organically modified clay [11].

The hardness value represents the capacity of material to resist to the local surface deformation. The enhancement in hardness by addition of the filler is potentially due to the increased confinement of the epoxy resin inside the clay galleries.

The values of elastic modulus obtained by nanoindentation tests are different from the values obtained by DMA measurements. The difference is due to the different experimental approaches between the two techniques. In particular as for the indentation analysis if the polymer is creeping while unloading, this would tend to increase the slope of the in the opposing to the epoxy macromolecular movements and enhances the hardness of the epoxy matrix.

\section{ACKNOWLEDGEMENTS}

The authors would like to thank the Mr. Mario De Angioletti and Mrs. Mariarosaria Marcedula their technical support for DSC and DMA tests, and Dr. Aniello Cammarano for his technical support for nano-indentation tests.

\section{REFERENCES}

[1] Osman, M.A.; Atallah, A.; Muller, M.; Suter, U.W. Reinforcement of poly(dimethylsiloxane) networks by mica flakes. Polymer, 2001, 42(15), 6545-6556.

[2] Ou, Y.; Yang, F.; Yu, Z.Z. A new conception on the toughness of nylon 6/silica nanocomposite prepared via in situ polymerization. $J$. Polym. Sci. Part B: Polym. Phys., 1998, 36(5), 789-795.

[3] He, H.; Duchet, J.; Galy, J.; Gerard, J-F. Grating of swelling clay materials with 3-aminoprpyltriethoxysilane. J. Colloid Interface Sci., 2005, 288(1), 171-176.

[4] Liu, Y.-L.; Hsu, C.-Y.; Wei, W.-L.; Jeng, R. Preparation and thermal properties of epoxy-silica nanocomposites from nanoscale colloidal silica. Polymer, 2003, 44(18), 5159-5167.

[5] Preghenella, M.; Pegoretti, A.; Migliaresi, C. Thermo-mechanical characterization of fumed silica-epoxy nanocomposites. Polymer, 2005, 46(26), 12065-12072.

[6] Liu, A.; Xie, T.; Yang, G. Synthesis of exfoliated monomer casting polyamide $6 / \mathrm{Na}^{+}$-montmorillonite nanocomposites by anionic 
ring opening polymerization. Macromol. Chem. Phys., 2006, 207(7), 701-707.

[7] Piscitelli, F.; Callegaro, G.; Lavorgna, M.; Amendola, E. Clay Functionalization with Different Aminosilanes for Nanocomposites Preparation, Proceedings of the 4th International Conference on Times of Polymers (TOP) and Composites, Ischia, Italy, September 21-24, 2008; Acierno, D.; D’Amore, A.; Grassia, L., Eds.; University of Naples Federico II: Naples, IT, 2008; pp. 181-183.

[8] Shanmugharaj, A.M.; Rhee, K.Y.; Ryu, S.H. Influence of dispersing medium of grafting of aminopropyltriethoxysilane in swelling clay materials. J. Colloid Interface Sci., 2006, 298(2), 854-859.
[9] Oliver, W.C.; Pharr, G.M. An improved technique for determining hardness and elastic-modulus using load and displacement sensing indentation experiments. J. Mater. Res., 1992, 7, 1564-1583.

[10] Galimberti, M.; Senatore, S.; Conzatti, L. Formation of clay intercalates with organic bilayers in hydrocarbon polymers. Polym. Adv. Technol., 2009, 20(2), 135-142.

[11] Shen, L.; Wang, L.; Liu, T. Nanoindentation and morphological studies of epoxy nanocomposites. Macromol. Mater. Eng., 2006, 291(11), 1358-1366.

[12] Zheng, S.; Ashcroft, I.A. A depth sensing indentation study of the hardness and modulus of adhesives. Int. J. Adhes. Adhes., 2005, 25(1), 67-76.

(C) Amendola et al.; Licensee Bentham Open.

This is an open access article licensed under the terms of the Creative Commons Attribution Non-Commercial License (http://creativecommons.org/licenses/by-nc/3.0/) which permits unrestricted, non-commercial use, distribution and reproduction in any medium, provided the work is properly cited. 